\title{
CORRELATED FERMIONS CLOSE TO MOTT LOCALIZATION: DEVIATIONS FROM LANDAU FERMI-LIQUID PICTURE
}

\author{
J. SPALEK ${ }^{a}$, W. WóJCiK ${ }^{b}$ AND P. KorbeL ${ }^{a}$ \\ anstitute of Physics, Jagiellonian University, Reymonta 4, 30-059 Kraków, Poland \\ ${ }^{b}$ Institute of Physics, Technical University, Podchorążych 1, 30-084 Kraków, Poland
}

We summarize the main novel features of almost localized fermions in the presence of an applied magnetic field: (i) the spin dependence of their effective mass, which leads to quantum beats in the de IIaas-van Alphen effect and (ii) the presence of a nonlincar molecular field and related metamagnetic behavior.

PACS numbers: 71.27.ta, 71.30.+h, 71.10.Fd

The Fermi liquid character of a metal close to the metal-insulator (Mott) boundary should not be taken for granted, since in those systems the Coulomb interaction energy is comparable to the band (and Fermi) energy of the relevant electrons [1]. In this communication we summarize briefly the specific properties of those fermions, which provide a test for their Fermi-liquid character. Most of these properties have been introduced earlier [2-4]; here we provide a physical discussion starting from the concepts of spin-split mass and of nonlinear molecular field, and provide a set of experimentally vcrifiable predictions.

A mean field theory in the Formi-liquid regime can be formulated easily within the slave boson approach [5]. In this approach the IIamiltonian $H-\mu N_{\mathrm{e}}$ is expressed as follows:

$$
\begin{aligned}
& I-\mu N_{\mathrm{e}}= \\
& \sum_{i j \sigma}^{\prime} t_{i j} z_{i \sigma}^{\dagger} z_{j \sigma} f_{i \sigma}^{\dagger} f_{j \sigma}+U \sum_{i} d_{i}^{\dagger} d_{i}-\mu \sum_{i \sigma} f_{i \sigma}^{\dagger} f_{i \sigma}-\mu_{\mathrm{B}} H_{a} \sum_{i \sigma} \sigma f_{i \sigma}^{\dagger} f_{i \sigma} .
\end{aligned}
$$

In this approach $z_{i \sigma}^{\dagger} \equiv e_{i} p_{i \sigma}^{\dagger}+d_{i}^{\dagger} p_{i \bar{\sigma}}$ expresses a correlated nature of the intersite lopping, other symbols are standard [5]. The dynamics of this Fermi-Bose system must respect three constraints at each site: $P_{i} \equiv e_{i}^{\dagger} e_{i}+\sum_{\sigma} p_{i \sigma}^{\dagger} p_{i \sigma}+d_{i}^{\dagger} d_{i}-1 \equiv 0$ and $Q_{i \sigma} \equiv f_{i \sigma}^{\dagger} f_{i \sigma}-\left(p_{i}^{\dagger} p_{i}+d_{i}^{\dagger} d_{i}\right) \equiv 0$. In the Lagrangian formalism, the constraints are expressed through the part $L_{c}(\tau)=\sum_{i} \lambda_{i}^{(1)} P_{i}+\sum_{i} \lambda_{i \sigma}^{(2)} Q_{i \sigma}$, so that the total Lagrangian reads $L(\tau)=L_{0}(\tau)+H(\tau)+L_{c}(\tau)$, where $L_{0}(\tau)=\sum_{i} e_{i}^{\dagger} \partial_{\tau} e_{i}+$ $\sum_{i \sigma} f_{i \sigma}^{\dagger} \partial_{\tau} f_{i \sigma}+\ldots$, represents the term with time derivatives of all involved Fermi 
and Bose operators. One should note that the present formulation contains spin-dependent Lagrange factörs $\lambda_{i \sigma}^{(2)}$, as our metallic state is magnetically polarized in the applied field.

A mean-field state corresponds to a condensed state of all bosons $\left(e, p_{\sigma}, d\right)$. Assuming additionally that the macro state is spatially homogeneous, the constraints are expressed via constants $\lambda_{i}^{(1)}=\lambda^{(1)}$ and $\lambda_{i \sigma}^{(2)}=\lambda_{\sigma}^{(2)}$. In effect, the free-energy functional assumes the form

$$
\begin{aligned}
& F / N=-k_{\mathrm{B}} T \frac{1}{N} \sum_{k_{\sigma}} \ln \left(1+\exp \left(-\frac{q_{\sigma} \epsilon_{k}-\left(\mu_{\mathrm{B}} H_{a}-\lambda_{\sigma}^{(2)}\right) \sigma-\mu}{k_{\mathrm{B}} T}\right)\right) \\
& +U d^{2}+\mu n+\lambda^{(1)}\left(e^{2}+d^{2}+p_{\uparrow}^{2}+p_{\downarrow}^{2}-1\right)+\lambda_{\uparrow}^{(2)}\left(p_{\uparrow}^{2}+d^{2}\right)+\lambda_{\downarrow}^{(2)}\left(p_{\downarrow}^{2}+d^{2}\right) .
\end{aligned}
$$

One should notice that the mean-field approximation for Bose fields leads to the fermionic quasiparticles with energy $E_{k_{\sigma}}=q_{\sigma} \epsilon_{k}-\left(\mu_{\mathrm{B}} H_{a}-\lambda_{\sigma}^{(2)}\right) \sigma$, where $q_{\sigma}$ is the spin-dependent band narrowing,

$$
q_{\sigma}=\frac{e^{2} p_{\sigma}^{2}+d^{2} p_{\bar{\sigma}}^{2}+2 e d p_{\sigma} p_{\vec{\sigma}}}{\left(1-d^{2}-p_{\sigma}^{2}\right)\left(1-e^{2}-p_{\bar{\sigma}}^{2}\right)}
$$

and $\lambda_{\sigma}$ is an effective (molecular) field acting on them. The factor $q_{\sigma}$ leads in a natural manner to the spin-dependent masses via the relation $m_{\sigma} / m_{0}=1 / q_{\sigma}$. Both $m_{\sigma}$ and $\lambda_{\sigma}$ are absent in the Landau Fermi liquid theory. These factors, together with the boson field amplitudes are determined by minimizing the functional (2) with respect to all parameters $\left(e, p_{\sigma}, d, \mu, \lambda^{(1)}\right.$, and $\left.\lambda_{\sigma}^{(2)}\right)$. This minimization reflects both a self-consistent adjustment of the balance between the single-particle (the first term) and the interaction $\left(U d^{2}\right)$ energies, and fixing the constraints on average scale. Below we discuss the results obtained by implementing the above procedure. A simple relation between the equations reduces the system of self-consistent equations to two: for the double occupancy probability $d^{2}$, and for the magnetization $m \equiv p_{\uparrow}^{2}-p_{\downarrow}^{2}$, respectively.

In Fig. 1a, b we have plotted respectively the quantities $d^{2}$ and $m$, both as a function of the reduced field $h \equiv \mu_{\mathrm{B}} H_{a} / W$. Note the metanagnetic transition

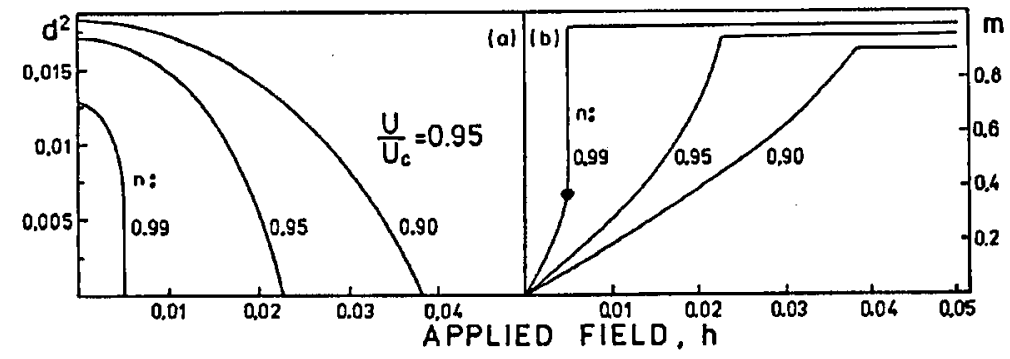

Fig. 1. Field dependent double occupancy (a) and magnetization (b) for three-band fillings. The solid circle on the curve for $n=0.99$ marks the metamagnetic transition. $U / U_{\mathrm{c}}=0.95$ is the relative magnitude of intratomic interaction. 


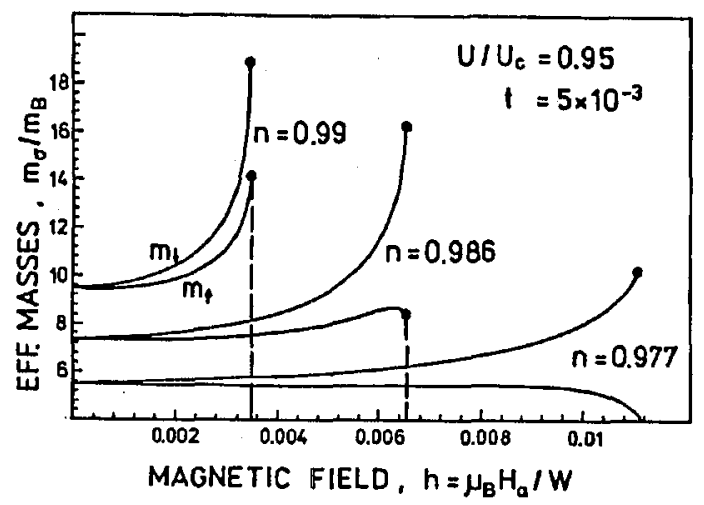

Fig. 2. Applied field dependences of the effective masses $m_{\uparrow}$ (majority spin) and $m_{\downarrow}$ for three-band fillings $n$ specified, $U / U_{\mathrm{c}}=0.95$, and reduced temperature $t \equiv k_{\mathrm{B}} T / W=5 \times 10^{-3}$.

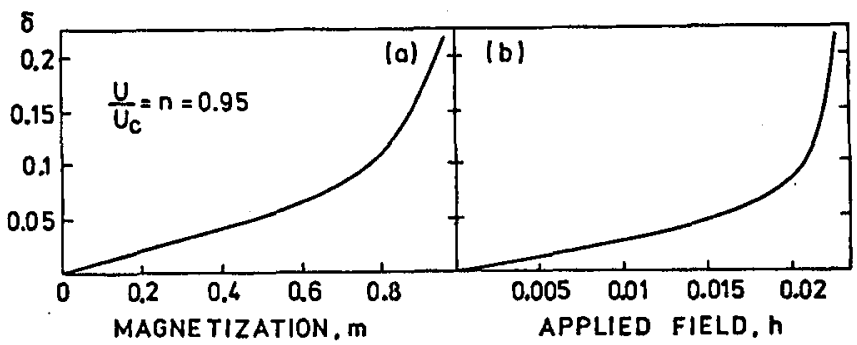

Fig. 3. The effective field $\delta$ as a function of magnetization (a) and of applied field $h \equiv \mu_{\mathrm{B}} H_{a} / W(\mathrm{~b})$.

for the band filling $n=0.99$, but only a metamagnetic behavior for the lower $n$, in accordance with the general phase diagram in Ref. [3]. The quantities displayed in Fig. 1 are used to determine other system properties. Explicitly, in Fig. 2 we have plotted the spin-split masses $\dot{m}_{\sigma} / m_{0}$ starting from a paramagnetic state in the field absence. The effective masses in the spin minority $(\downarrow)$ subband grow and this growth terminates at the point, where the system saturates magnetically. Such dependence will lead to a maximum in the field dependence of the linear specific heat coeflicient $\gamma \propto\left(m_{\uparrow}+m_{\downarrow}\right)$. The quasiparticles in the majority spin subband acquire the bare band value at that point.

To determine the character of the spin-splitting we have plotted in Fig. 3a, b the magnetization (a) and field (b) dependences of the spin-splitting $\delta=\left|\lambda_{\uparrow}^{(2)}-\lambda_{\dagger}^{(2)}\right| / W$. This provides us with information about the nonlinearity of the effective field. The nonlinearity bears its origin in the field dependence of the effective mass.

The eflective masses are determined directly from the de Haas-van Alphen magnetization oscillations in the field. In Fig. 4 we provide those oscillation in the lower fields, i.e. away from the metamagnetic transition. In that situation the 


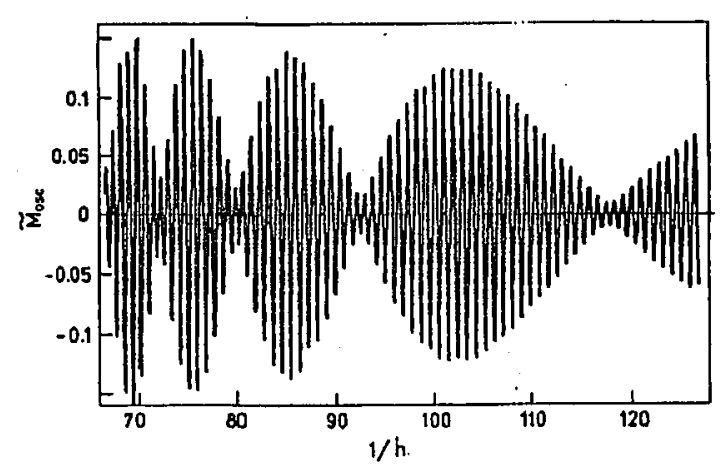

Fig. 4. The amplitude of the de IIaas-van Alphen oscillations as a function of inverse field. The parameters are $U / U_{\mathrm{c}}=n=0.95$.

small difference between $m_{\uparrow}$ and $m_{\downarrow}$ will produce the spectacular quantum beats reflected in the frequency variation with the field. Those beats should disappear when the system approaches magnetic saturation.

In summary, the concept of almost localized Fermi liquid leads both to the spin-split effective masses and to the strong and nonlinear molecular field. Those quantities were introduced and discussed on the mean field level. It is important to determine those properties experimentally to verify whether the mean-field approach is a good starting point to the metallic systems close to the Mott-IIubbard localization boundary. Only then the theoretical considerations involving quantum (Gaussian) fluctuations will represent the next step in approaching those metals in a systematic manner.

The paper was supported by the Committee for Scientific Research through grant No. 2P302 09305.

\section{References}

[1] For a review of this point see: J. Spałek, J. Sol. Slate Chem. 88, 70 (1990).

[2] J. Spałek, W. Wójcik, in: Spectroscopy of the Molt Insulators and Correlated Metals, Eds. A. Fujimori, Y. Tokura, in series Springer Tracts in Solid State Sciences, Vol. 119, Springer, Berlin 1995, p. 41.

[3] P. Korbel, J. Spałek, W. Wójcik, Phys. Rev. B 52, R2213 (1995); J. Spalek, P. Gopalan, Phys. Rev. Lett. 64, 2823 (1990).

[4] J. Spałek, W. Wójcik, J. Magn. Magn. Mater. 140-144, 1199 (1995).

[5] For review see: R. Frésard, P. Wölhe, Int. J. Mod. Phys. B 6, 685 (1992); M. Lavagna, Phys. Rev. B 41, 142 (1990). 